\title{
Wall Stress and Patterns of Hypertrophy in the Human Left Ventricle
}

\author{
William Grossman, Donald Jones, and Lambert P. McLaurin \\ From the C. V. Richardson Cardiac Catheterization Laboratory and the \\ Department of Medicine, University of North Carolina School of Medicine, \\ Chapel Hill, North Carolina 27514
}

A B S T R A C T It is generally recognized that chronic left ventricular (LV) pressure overload results primarily in wall thickening and concentric hypertrophy, while chronic LV volume overload is characterized by chamber enlargement and an eccentric pattern of hypertrophy. To assess the potential role of the hemodynamic factors which might account for these different patterns of hypertrophy, we measured LV wall stresses throughout the cardiac cycle in 30 patients studied at the time of cardiac catheterization. The study group consisted of 6 subjects with LV pressure overload, 18 with LV volume overload, and 6 with no evidence of heart disease (control). LV pressure, meridional wall stress $\left(\sigma_{m}\right)$, wall thickness $(h)$, and radius $(R)$ were measured in each patient throughout the cardiac cycle. For patients with pressure overload, LV peak systolic and end diastolic pressures were significantly increased (220 $\pm 6 /$ $23 \pm 3 \mathrm{~mm} \mathrm{Hg})$ compared to control $(117 \pm 7 / 10 \pm 1 \mathrm{~mm}$ $\mathrm{Hg}, P<0.01$ for each). However, peak systolic and end diastolic $\sigma_{m}$ were normal $\left(161 \pm 24 / 23 \pm 3 \times 10^{3} \mathrm{dyn} /\right.$ $\left.\mathrm{cm}^{2}\right)$ compared to control $\left(151 \pm 14 / 17 \pm 2 \times 10^{3} \mathrm{dyn} /\right.$ $\left.\mathrm{cm}^{2}, \mathrm{NS}\right)$, reflecting the fact that the pressure overload was exactly counterbalanced by increased wall thickness $(1.5 \pm 0.1 \mathrm{~cm}$ for pressure overload vs. $0.8 \pm 0.1 \mathrm{~cm}$ for control, $P<0.01)$. For patients with volume overload, peak systolic $\sigma_{m}$ was not significantly different from control, but end diastolic $\sigma_{m}$ was consistently higher than normal $\left(41 \pm 3 \times 10^{3} \mathrm{dyn} / \mathrm{cm}^{2}\right.$ for volume overload, $17 \pm 2 \times 10^{3} \mathrm{dyn} / \mathrm{cm}^{2}$ for control, $\left.P<0.01\right)$. LV pressure overload was associated with concentric hypertrophy, and an increased value for the ratio of wall thickness to radius ( $h / R$ ratio). In contrast, $\mathrm{LV}$ volume

Dr. McLaurin is the recipient of Special Research Fellowship 1-F03-HL 55243-01 from the National Heart and Lung Institute.

Received for publication 22 October 1974 and in revised form 14 March 1975. overload was associated with eccentric hypertrophy, and a normal $h / R$ ratio. These data suggest the hypothesis that hypertrophy develops to normalize systolic but not diastolic wall stress. We propose that increased systolic tension development by myocardial fibers results in fiber thickening just sufficient to return the systolic stress (force per unit cross-sectional area) to normal. In contrast, increased resting or diastolic tension appears to result in gradual fiber elongation or lengthening which improves efficiency of the ventricular chamber but cannot normalize the diastolic wall stress.

\section{INTRODUCTION}

It is generally assumed that hypertrophy of cardiac muscle is a useful physiologic adaptation which develops when an increased work load is chronically imposed on the myocardium (1). Although the hypertrophied myocardium may allow maintenance of adequate cardiac compensation for many years, eventually it becomes incapable of meeting the increased work load imposed upon it, and heart failure ensues. For this reason, it has been suggested that myocardial hypertrophy may be considered the interface between the normal and failing heart (2).

Before heart failure occurs, hypertrophy has generally become well developed and moreover it appears to have developed in a pattern unique to the inciting stress. Thus concentric hypertrophy is seen in aortic stenosis and hypertension $(1,3-5)$, eccentric ${ }^{1}$ hypertrophy in aortic or mitral insufficiency $(1,3)$, and asymmetric hypertrophy in idiopathic hypertrophic subaortic stenosis (6). Although factors which may act as stimuli to cardiac hypertrophy have been extensively studied in

\footnotetext{
${ }^{1}$ So termed because the cavity enlarges laterally in the chest, and becomes eccentric with regard to its normal position (4).
} 
the experimental animal (2, 7-10), little is known about factors which influence the pattern of hypertrophy in either animals or man $(3-5,11)$. The present study was undertaken to examine the role of alterations in diastolic and systolic wall stresses in influencing the pattern and extent of left ventricular (LV) ${ }^{2}$ hypertrophy in man.

\section{METHODS}

30 patients undergoing complete left and right heart catheterization for diagnostic purposes formed the study population. Included were 18 patients with $\mathrm{LV}$ volume overload, 6 patients with pressure overload, and 6 patients with no hemodynamic or angiographic evidence of heart disease, who served as normal controls. Diagnostic and hemodynamic data for each patient are detailed in Table I. At the time of cardiac catheterization, all patients were well compensated with regard to congestive heart failure, with the exception of volume overload patients 4, 7, and 12, whose data are given in Table I. Patients 4 and 12 had persistent severe orthopnea and paroxysmal nocturnal dyspnea despite digitalis and diuretic treatment, and patient 7 was decompensated from acute aortic insufficiency secondary to bacterial endocarditis.

Catheterization was carried out in the fasting state after diazepam (5-10 mg i.m.) premedication. Brachial arteriotomy and retrograde left heart catheterization were performed with standard no. 8 French catheters in 18 patients, and with high fidelity micromanometer-tipped catheters (Mikrotip, Millar Instruments, Houston, Tex.) in 12 patients. Frequency response characteristics of the standard fluid-filled catheter system used in our laboratory have been previously reported (12); the undamped natural frequency is $31 \mathrm{~Hz}$, and the damping system used gives a measured damping coefficient of 0.603 , indicating a frequency response flat to approximately $25 \mathrm{~Hz}$. Zero reference for pressure measurement was taken as the midchest with the patient supine. LV cineangiography was performed in each study, and patients with regional abnormalities of contraction were excluded.

Immediately before angiography a simultaneous strip chart recording of electrocardiogram (ECG), LV pressure, and ultrasonically determined septal and posterior wall motion (Fig. 1) in the plane of the mitral valve (recorded with an Ekoline 20-A ultrasonoscope [Smith Kline Instruments, Palo Alto, Calif.] interfaced to a DR-12 recorder [Electronics for Medicine, Inc., White Plains, N. Y.]) was obtained as previously described from this laboratory (13-16). The use of pulsed reflected ultrasound to measure LV dimensions and wall thickness in man has been validated now by several investigators (17-23), and is discussed in detail in the Discussion section. In this study, the LV internal diameter, $D$, was measured as the vertical distance between echoes from the endocardial surfaces of the interventricular septum and LV posterior wall, and posterior wall thickness, $h$, was measured as the distance between endocardial and epicardial echoes on the ultrasonic tracing (Fig. 1). LV mass index was estimated for each patient from $D$ and $h$ according to the method of Troy, Pombo, and Rackley (24). This method has been reported

\footnotetext{
2 Abbreviations used in this paper: ECG, electrocardiogram; $h$, left ventricular wall thickness; LV, left ventricular; $\sigma_{m}$, meridional wall stress.
}

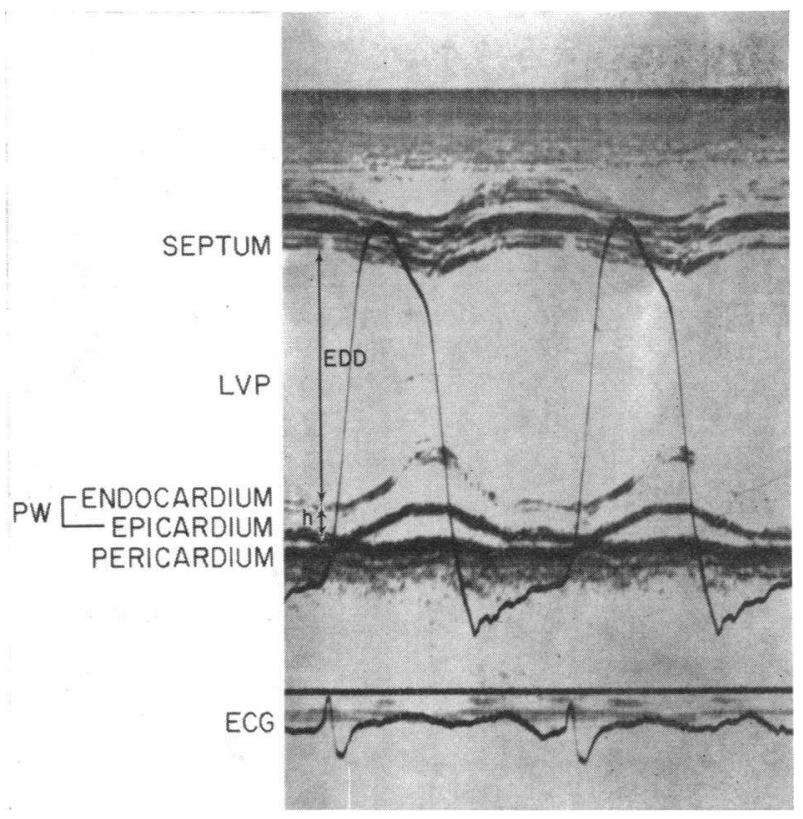

FIgURE 1 Strip chart recording of LV pressure (LVP) and simultaneous ultrasonic tracing of LV septum and posterior wall $(\mathrm{PW})$ endocardium and epicardium in a patient with mitral insufficiency around the base of a prosthetic valve. LV chamber diameter is measured as the distance between septal and PW endocardial surfaces, and the end diastolic diameter is illustrated as EDD. LV wall thickness, $h$, is measured as the distance between PW endocardial and epicardial surfaces. See text for details of technique. LVP is measured with a micromanometer-tipped catheter.

by them to show excellent correlation with LV mass calculated from biplane LV angiograms.

Theoretic considerations and calculations. LV wall stress is a function of chamber size and configuration, thickness of the ventricular wall, and intraventricular pressure. For either an ellipsoidal or a spherical model, an average meridional stress $\left(\sigma_{m}\right)$ can be defined (Fig. 2) as the force per unit area acting at the midplane to the heart, in the direction of the apex to base length (25). As seen in Fig. 2 this may be derived by equating the meridional wall forces $\left(\sigma_{m} \times \pi\left[R_{o}{ }^{2}-R_{i}{ }^{2}\right]\right)$ to the pressure loading $\left(P \pi R_{i}{ }^{2}\right)$, since these must be exactly equal if the ventricle is to hold together. Thus,

$$
\begin{gathered}
\sigma_{m} \times \pi\left(R_{o}{ }^{2}-R_{i}{ }^{2}\right)=P \pi R_{i}{ }^{2}, \\
\left.\sigma_{m}=P R_{i}{ }^{2} / R_{o}{ }^{2}-R_{i}{ }^{2}\right),
\end{gathered}
$$

and if $\left(R_{o}-R_{i}\right)=h$, the wall thickness, then

$$
\sigma_{m}=\frac{P R_{i}{ }^{2}}{\left(R_{o}-R_{i}\right)\left(R_{o}+R_{i}\right)}=\frac{P R_{i}}{2 h\left(1+h / 2 R_{i}\right)} .
$$

Thus, an average meridional or longitudinal stress may be calculated throughout the cardiac cycle from the combined ultrasonic and hemodynamic data described above which make available simultaneous LV pressure, wall thickness $(h)$, and radius $(D / 2)$. It should be pointed out that there are two stresses acting at the equatorial plane illustrated in Fig. 2; the meridional stress, acting parallel to the long 


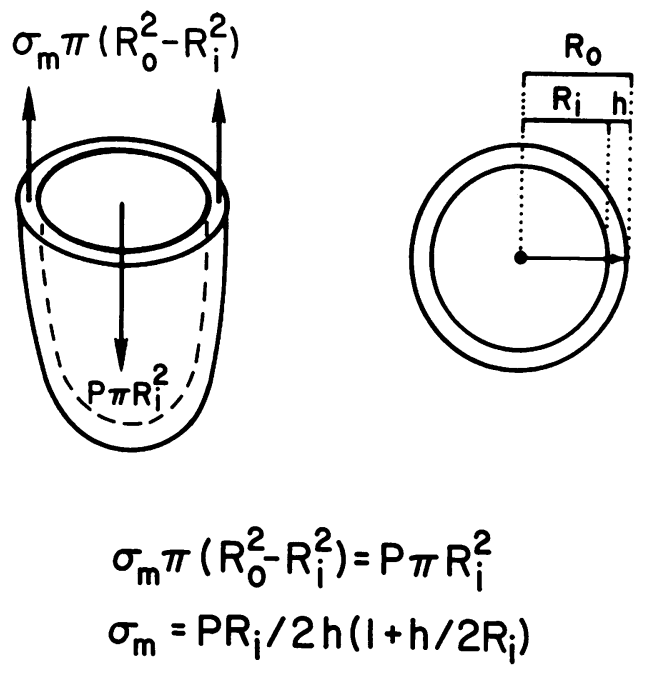

FIGURE 2 Diagrammatic representation of an idealized LV chamber in coronal section, looking from the front (left) and above (right). Wall thickness $(h)$, inner radius $\left(R_{i}\right)$, and outer radius $\left(R_{o}\right)$ are required to calculate meridional wall stress $\left(\sigma_{m}\right)$. This is accomplished by equating the meridional wall forces $\left(\sigma_{m} \times \pi\left[R_{o}{ }^{2}-R_{i}{ }^{2}\right]\right)$ to the pressure loading $\left(P \pi R_{\mathfrak{t}}{ }^{2}\right)$, since these must be exactly equal if the ventricle is to hold together. The same calculation applies for either an ellipsoidal or a spherical model. See text for details.

axis, and the circumferential stress acting perpendicular to the long axis. For a spherical model, the two are equal, while for an ellipsoidal model the circumferential stress tends to be larger than the meridional stress.

LV meridional stress $\left(\sigma_{m}\right)$ was calculated throughout the cardiac cycle in each of the 30 subjects by using Eq. 3 and values for $P, h$, and $R$, obtained at 20 -ms intervals. End diastolic measurements were taken at the time of the peak of the $R$ wave of the ECG, while peak systolic $\sigma_{m}$ was determined as the highest value for $\sigma_{m}$ occurring in systole. It should be emphasized that peak systolic $\sigma_{m}$ usually occurred early in ejection (within 40-60 ms of aortic valve opening) and was quite different from end systolic $\sigma_{m}$, which was of much smaller magnitude. Mean systolic $\sigma_{m}$ was also determined for each subject by averaging values for $\sigma_{m}$ from end diastole to end systole. Measurements were averaged over at least 3-5 beats for each patient, and over an entire respiratory cycle if significant respiratory variation in pressures was observed. Statistical analysis was carried out by using the standard unpaired $t$ test for analysis of variance (26).

Validity of the method. To validate the stress measurements made by this combined hemodynamic-ultrasonic technique, we measured meridional stress using angiographic data throughout the cardiac cycle in representative normal subjects as well as those with volume overload or pressure overload of the left ventricle (27). Angiographic wall thickness, long axis, and minor axis were measured from LV cineangiograms taken in the right anterior oblique projection, and corrected for magnification by use of a calibrated grid. Meridional wall stress was calculated by using Eq. 3 above. It should be recognized that minor axis and wall thickness measured by echocardiogram best correspond anatomically to those seen in a left anterior oblique angiogram. Agreement of echocardiographic and angiographic measurements therefore depends not only on the validity of both methods, but on the presence of ventricular symmetry with regard to minor axis and wall thickness. Good agreement of the methods was observed, with correlation coefficients for echocardiographic and angiographic measurements averaging 0.91 for diameters, 0.81 for wall thickness, and 0.96 for meridional wall stress (27). An example of wall stress measurements throughout the cardiac cycle in a representative normal subject is shown in Fig. 3. Although the angiographic data was used as a reference standard, we feel that it is important to point out that the stress calculations made by using the hemodynamic-ultrasonic technique may be superior in their physiologic accuracy to the so called "reference" standard. First, the hemodynamic and ultrasonic data are obtained simultaneously (Fig. 1), allowing truly instantaneous pressure-diameterwall thickness measurements for calculation of stress. For the angiographic data, diameter and wall thickness measurements from the left ventriculogram are matched up with pressure measurements made at an earlier time. Slight variations in cycle length, ejection time, and systolic and diastolic LV pressures may significantly affect the comparability of these measurements. Furthermore, the injection of $30-50 \mathrm{~cm}^{3}$ of viscous contrast media into a $\mathrm{LV}$ chamber containing $80-100 \mathrm{~cm}^{3}$ of blood may alter LV geometry, volume, and wall stresses in an unknown and unpredictable fashion. None of these particular deficiencies apply to the ultrasonic technique, and we feel that these considerations

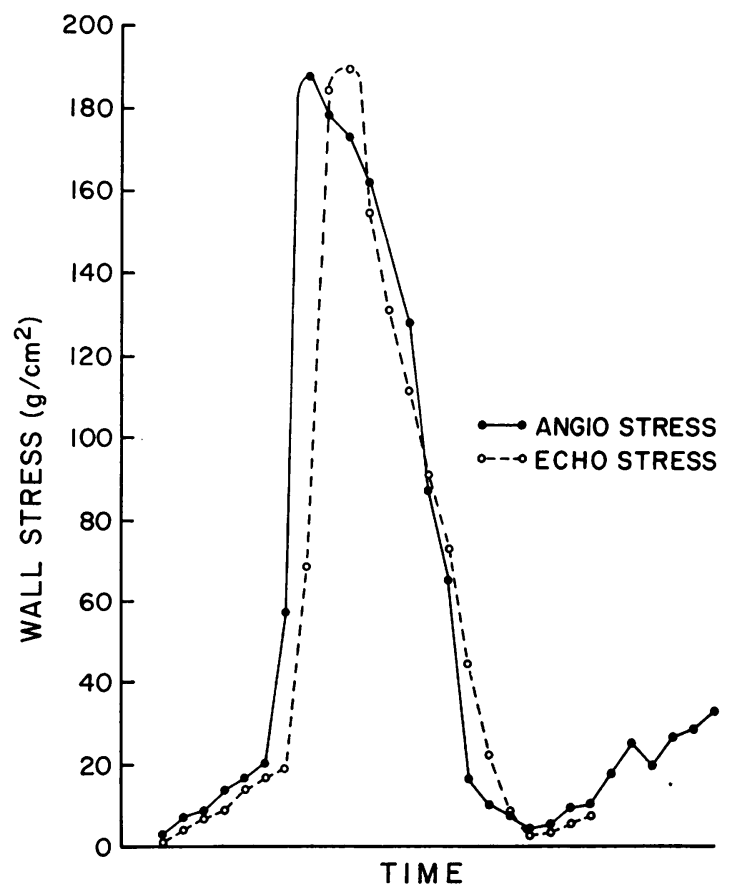

FIgURE 3 Meridional wall stress calculated throughout the cardiac cycle in a normal subject from simultaneous measurements of LV pressure, wall thickness, and minor axis. Wall stress calculated from angiographic (angio) and ultrasonic (echo) data show excellent agreement with close superimposition of stress-time plots constructed by the different methods. Similar agreement was seen for pressure and volume overload ventricles (27). 
support the usefulness of the combined hemodynamic-ultrasonic approach described herein.

\section{RESULTS}

Data relating to diagnosis, hemodynamics, wall stress, and LV chamber dimensions are summarized in Table I. As can be seen, LV mass index was more than twice normal for both the pressure and volume overload groups, indicating significant hypertrophy. Wall thickness was significantly increased for both pressure and volume overload groups, but it was disproportionately increased in the pressure overloaded ventricles, indicating the presence of concentric hypertrophy. This is borne out by examination of the $h / R$ ratio (Table I, Fig. 4), which is increased in patients with pressure overload, but normal in the volume overloaded ventricles, indicating concentric hypertrophy in the former as opposed to an eccentric or magnification pattern of hypertrophy in the latter group. It is interesting to note that the $h / R$ ratios at end diastole and at peak systolic stress were quite similar (Fig. 4).

The time-course of changes in LV pressure, wall thickness, and $\sigma_{m}$ is shown in Fig. 5 for representative normal, pressure overloaded, and volume overloaded ventricles. Peak systolic $\sigma_{m}$ consistently occurred early in ejection, generally within $80-120 \mathrm{~ms}$ of the onset of ventricular systole, at a time when significant wall

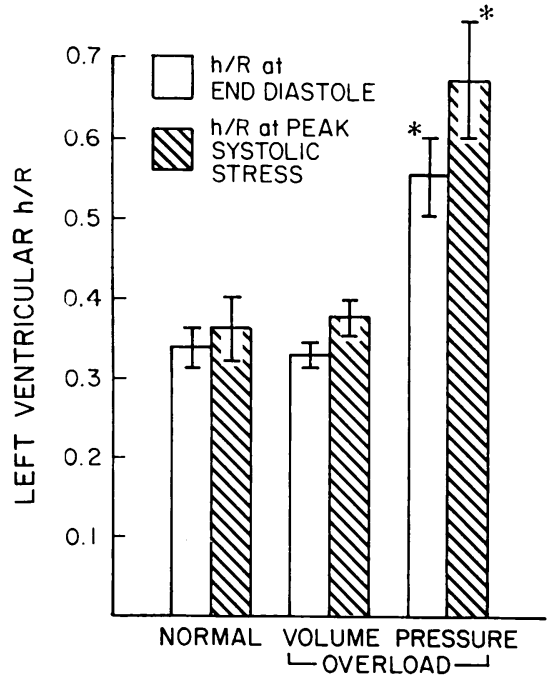

FIGURE 4 Ratio of LV wall thickness to internal radius $(h / R)$ at end diastole (clear bars) and at the time of peak systolic stress (hatched bars). $h / R$ is normal in patients with LV volume overload, but significantly increased in patients with pressure overload. Bars and brackets represent mean values and their standard errors, respectively. See text for details. $* P<0.001$ compared to normal.

thickening had not yet occurred. In contrast, at end ejection when wall thickness was maximum it can be
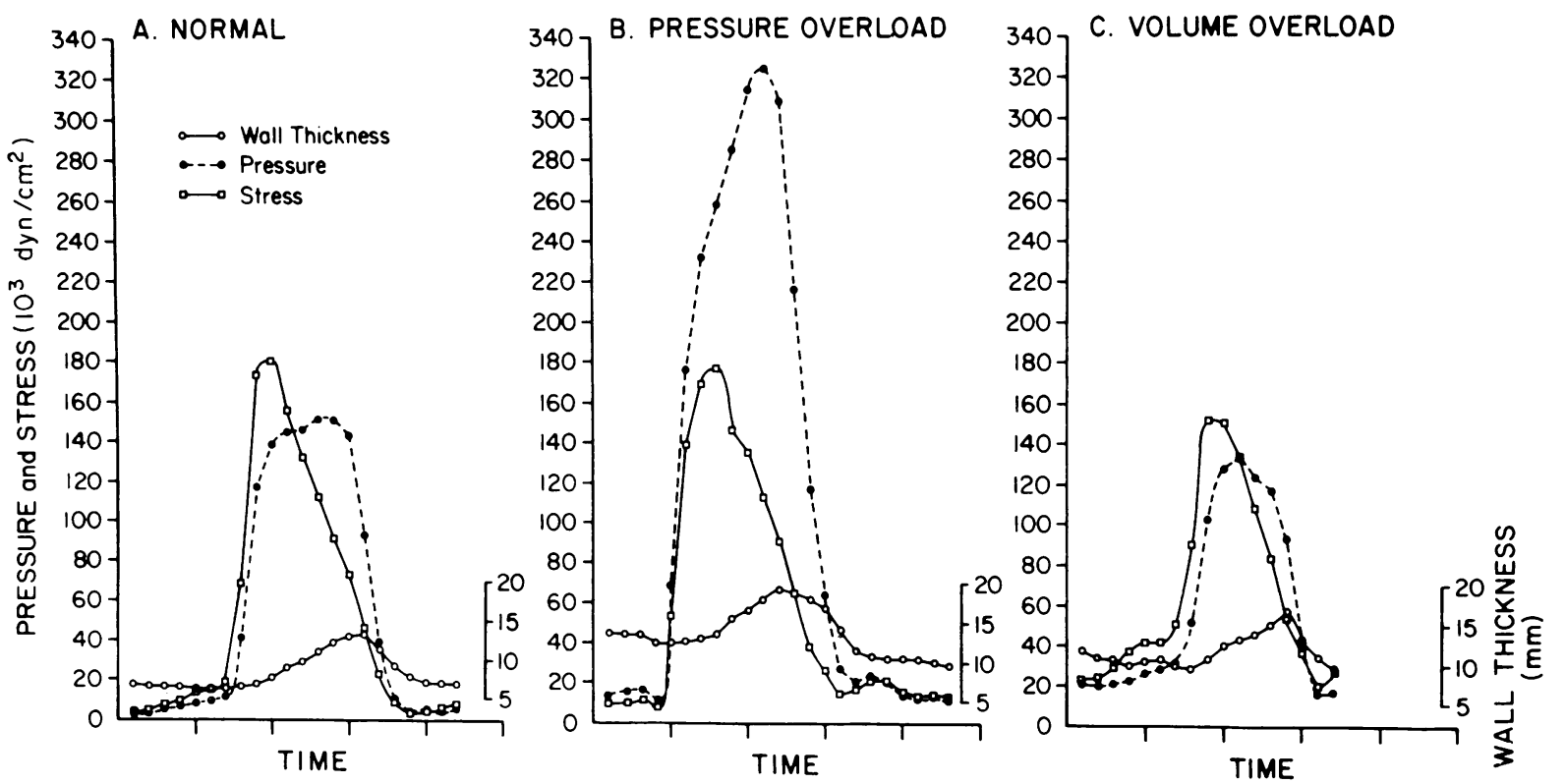

Figure 5 A comparison of changes in LV pressure (solid dots), wall thickness (open dots), and meridional stress (open squares) throughout the cardiac cycle for representative normal, pressure-overloaded, and volume-overloaded left ventricles. Measurements are plotted here at 40-ms intervals. In the pressure-overloaded ventricle (B), the markedly elevated systolic pressure is exactly counterbalanced by increased wall thickness with the result that wall stress remains normal. In the volume-overloaded ventricle $(C)$, peak systolic stress is normal but end diastolic stress is significantly increased. See text for details. 


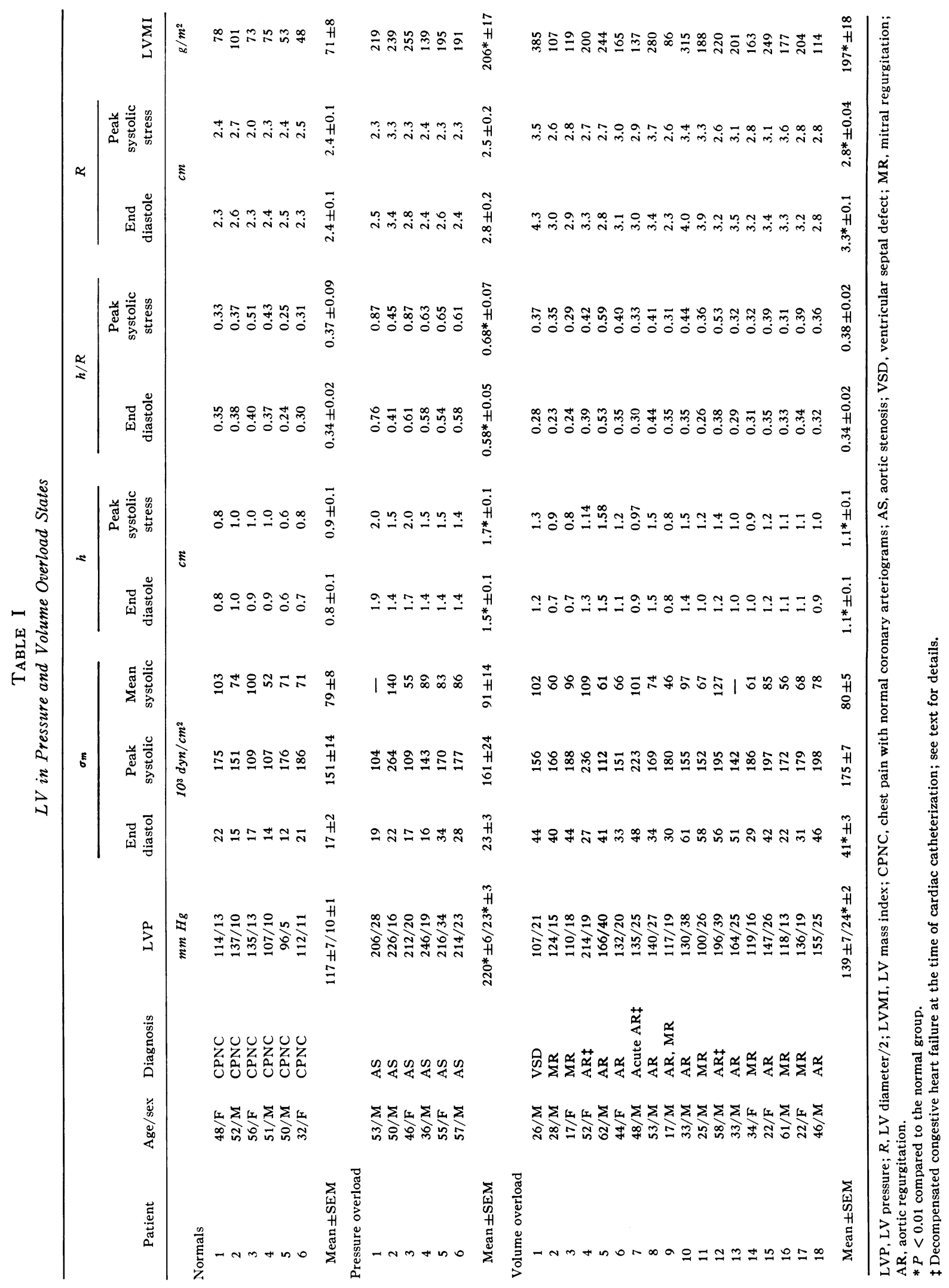


seen that $\sigma_{m}$ had declined substantially, although LV pressure was at or near peak levels.

LV peak and mean systolic stress were remarkably similar for all three groups (Table I, Fig. 6). There was no significant difference between either the pressure overload or volume overload group and normal, although peak systolic stress tended to be highest in those with volume overload. As mentioned above, the 18 patients with volume overload included three patients (4, 7, and 12 in Table I) with decompensated congestive failure at the time of study. Patients 4 and 12 had persistent severe orthopnea and paroxysmal nocturnal dyspnea despite digitalis and diuretics, while patient 7 had acute, recent onset aortic insufficiency due to bacterial endocarditis. In general, wall stress tended to be

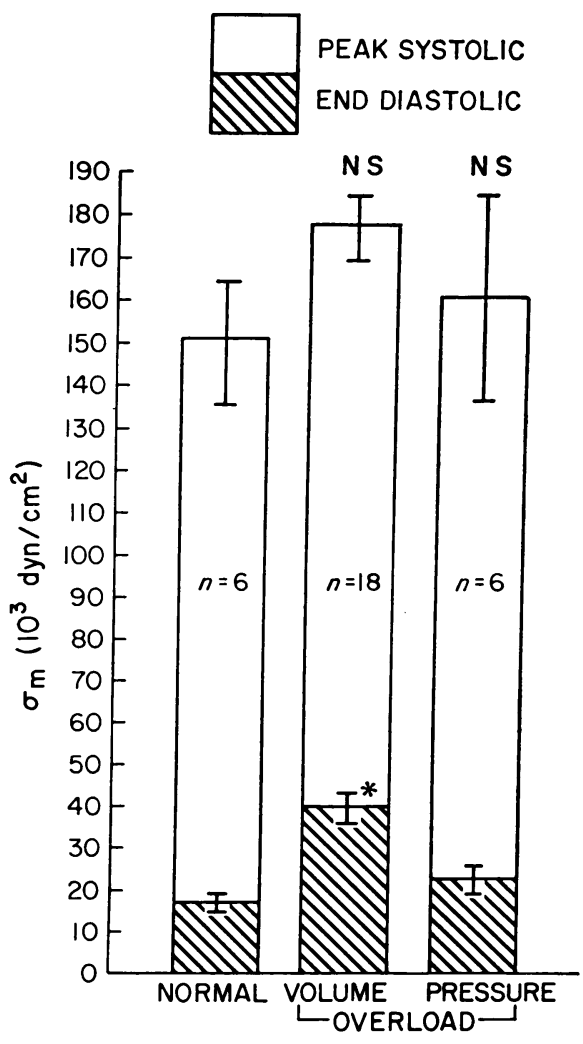

Figure 6 End diastolic and peak systolic meridional wall stress $\left(\sigma_{m}\right)$ in patients with LV volume overload, pressure overload, and normals. There is no significant difference in peak systolic $\sigma_{m}$ between either the pressure or volume overload group and normal. Peak systolic $\sigma_{m}$ tended to be highest in those with LV volume overload, reflecting the inclusion in this group of patients with decompensation or acute onset of the volume overload, as discussed in the text. In contrast to peak systolic $\sigma_{m}$, end diastolic $\sigma_{m}$ was consistently and significantly increased in patients with LV volume overload, and this appeared unrelated to decompensation or the recency of onset of the vclume overload. See text for discussion. $* P<0.01$.
HYPOTHESIS

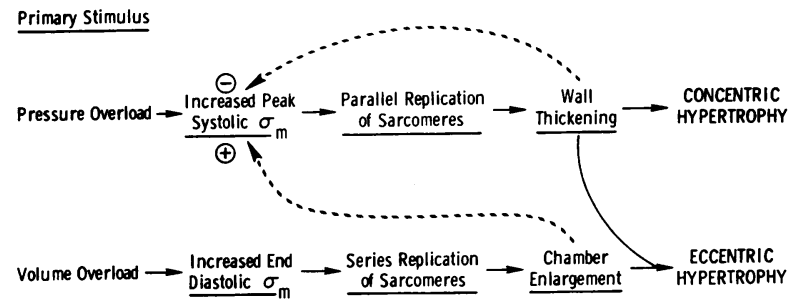

FIGURE 7 Hypothesis relating wall stress and patterns of hypertrophy. See text for details.

highest in these decompensated patients (Table I), and if they are excluded peak systolic wall stress for the volume overload group $\left(167 \pm 6 \times 10^{3} \mathrm{dyn} / \mathrm{cm}^{2}\right)$ more closely approximates that seen in the control subjects.

In contrast to peak and mean systolic stresses, end diastolic wall stress was consistently and significantly elevated in patients with volume overload as compared with either pressure overload or normal (Table I, Fig. $6)$. Further, the level of end diastolic stress appeared unrelated to decompensation, the recency of onset of volume overload, wall thickness, or the extent of overall hypertrophy as judged by the LV mass index.

As expected, LV peak systolic pressure was significantly increased for the pressure overload group (220 \pm 6 $\mathrm{mm} \mathrm{Hg}, P<0.01$ ), but not for the volume overload group (139 $\pm 7 \mathrm{~mm} \mathrm{Hg}, \mathrm{NS}$ ) as compared to normal $(117 \pm 7 \mathrm{~mm} \mathrm{Hg})$. LV end diastolic pressure was significantly increased for both pressure overload (23 \pm 3 $\mathrm{mm} \mathrm{Hg}, P<0.001)$ and volume overload $(24 \pm 2 \mathrm{~mm}$ $\mathrm{Hg})$ patients as compared to normal $(10 \pm 1 \mathrm{~mm} \mathrm{Hg})$, indicating the severity of the lesions in both groups.

\section{DISCUSSION}

Linzbach (3) believed that the most important factor controlling cardiac hypertrophy is the systolic force or tension generated by the myocardial fibers. His observations on autopsy specimens suggested that the pattern of hypertrophy is related to the inciting stress, with pressure overload leading to concentric hypertrophy and volume overload to eccentric hypertrophy. Sandler and Dodge (28) reported that systolic wall stresses in two patients with aortic stenosis were not higher than those seen in other patients in their study, and they speculated that the heart may hypertrophy to maintain wall stress within certain limits. Their study did not include sufficient numbers of patients with LV pressure overload, volume overload, and normal hemodynamics for meaningful intergroup comparisons. Hood, Rackley, and Rolett (29) investigated the relationship between wall stress and hypertrophy in a large group of 
patients and found that peak systolic stress was not significantly greater than normal in 18 patients with compensated volume overload and 1 patient with compensated pressure overload, but was increased in 4 patients with decompensated pressure or volume overload. Hood subsequently reported a small but statistically significant increase in peak systolic wall stress in 9 patients with compensated pressure overload (30). Grant, Greene, and Bunnell (4) examined LV chamber size and wall thickness in 5 patients with LV pressure overload and 10 with volume overload. Their data indicated that eccentric hypertrophy is analogous to the normal growth process by which a neonatal left ventricle is converted into the adult chamber, maintaining relative wall thickness constant. They emphasized the importance of the $h / R$ ratio, and although wall stress was not measured in their study, they suggested that in either pressure or volume overload the pattern of LV hypertrophy "tended to restore the cardiac myofibrils to their normal working conditions of tension and length" (4).

In our study, differences in the pattern of hypertrophy were clearly evident. Pressure overloaded left ventricles showed concentric hypertrophy as indicated by the increased $h / R$ ratio (Fig. 4 ), while volume-overloaded ventricles showed eccentric hypertrophy with an increased diameter but normal $h / R$ ratio (Table I, Fig. $4)$. Peak systolic wall stress was similar in normal, pressure-overloaded, and volume-overloaded left ventricles, and became nearly identical if patients with decompensated or recent onset, acute volume overload were excluded from analysis. In contrast to peak systolic stress, end diastolic stress was consistently and significantly increased in patients with volume overload, independent of the level of compensation or the recency of onset of the mechanical lesion. These observations are consistent with the concept previously advanced by others $(1,4,28,29)$ that in response to a chronic stress myocardial hypertrophy develops in such a manner as to maintain peak systolic wall stress within normal limits.

Experimental support for the importance of systolic ventricular wall tension as a stimulus to hypertrophy has been presented by Meerson (7) and others $(2,8)$. Peterson and Lesch (9) found that both active or "developed" tension and passive or resting tension served as stimuli to protein synthesis in the rabbit papillary muscle. In contrast, $\mathrm{Hjalmerson}$ and Isaksson found no effect on the rate of protein synthesis of the isolated working rat heart preparation when LV preload alone was increased (10). In our study, resting or diastolic stress remained high when volume overload was the principal cause of hypertrophy.

To account for these observations, we propose the following hypothesis (Fig. 7). When the primary stimulus to hypertrophy is LV pressure overload, we suggest that the resultant acute increase in peak systolic wall stress leads to parallel replication of sarcomeres, wall thickening, and concentric hypertrophy. The wall thickening is just sufficient to return peak systolic stress to normal, thus acting as a feedback inhibition. In contrast, when the primary stimulus is LV volume overload, increased end diastolic wall stress leads in this scheme to series replication of sarcomeres, fiber elongation and chamber enlargement, and eccentric hypertrophy (Fig. 7). It should be noted that chamber enlargement will lead acutely to increased peak systolic wall stress (by the Law of LaPlace), which in the postulated system then causes wall thickening of sufficient magnitude to normalize the systolic stress. Wall thickening then, as well as fiber elongation, contributes to the pattern of eccentric hypertrophy and this accounts for the observation in our patients that volume overload was associated with comparable increases in both wall thickness and radius so that their ratio, $h / R$, remained normal. A potential mechanism for the series replication of sarcomeres essential to this scheme might be found in the recent observations of Legato (31) concerning sarcomerogenesis by expansion and differentiation of the $Z$ substance. In our concept, the eccentric hypertrophy by which a child's heart becomes that of an adult represents a physiologic "volume overload," and may very well utilize the same mechanisms as seen with the pathologic volume overload of valvular insufficiency. One important shortcoming, however, is that we have examined an average wall stress across the entire thickness of the LV wall. More appropriate to the study of mechanisms of hypertrophy would be assessment of a "fiber-corrected stress," such as has been recently described by Falsetti, Mates, Grant, Greene, and Bunnell (32).

This study confirms Grant's observations (4) on the importance of the $h / R$ ratio. He pointed out that pure eccentric hypertrophy may be viewed as a "magnification" type of enlargement, characterized by chamber enlargement without alteration in relative wall thickness, while pure concentric hypertrophy is characterized by increased relative wall thickness, without significant chamber enlargement. Levine, Rockoff, and Braunwald (5) examined the ratio of $\mathrm{LV}$ diameter to wall thickness (the inverse of our $h / R$ ratio) and noted that this ratio was distinctly low in patients with $L V$ pressure overload, but in volume overload "did not differ in any consistent fashion from those observed in patients without ventricular disease." The $h / R$ ratio is thus relevant to the pattern and appropriateness of hypertrophy and has the great advantage that it can be noninvasively determined and followed in a given patient by the ultrasonic techniques utilized in this study. 
In support of the ultrasonic approach for measuring LV chamber dimensions, it should be pointed out that ultrasonic techniques have been extensively utilized to measure LV dimensions in experimental animals (33) and in man (17-23). Recently, Ratshin, Rackley, and Russell have reported (34) on the use of a combined hemodynamic and ultrasonic technique, similar to that used in our study, for the measurement of $\mathrm{LV}$ wall stress. In their study close correlation was demonstrated between wall stresses calculated from biplane angiographic and ultrasonic techniques. To validate the wall stress, wall thickness, and diameter measurements made in our own laboratory by the combined hemodynamic-ultrasonic technique, we measured meridional stress wall thickness, and minor axis using angiographic data throughout the cardiac cycle in representative normal, pressure overload, and volume overload subjects. The results (Fig. 3) showed an excellent correlation of the two methods (27). In fact, the present method for measuring meridional stress may have greater physiologic accuracy than the traditional angiographic method, for the reasons discussed above in the Methods section of this report.

In conclusion, the pattern and extent of LV hypertrophy were examined in 30 patients studied during cardiac catheterization. LV pressure overload was associated with concentric hypertrophy, normal peak systolic and end diastolic wall stresses, and an increased $h / R$ ratio. $\mathrm{LV}$ volume overload was associated with eccentric hypertrophy, increased end diastolic wall stress, normal $h / R$ ratio, and in general normal peak systolic wall stress. On the basis of these observations, an hypothesis is proposed relating wall stress and patterns of hypertrophy.

\section{ACKNOWLEDGMENTS}

This work was supported in part by U. S. Public Health Service grant HL 14883.

\section{REFERENCES}

1. Badeer, H. S. 1964. Biological significance of cardiac hypertrophy. Am. J. Cardiol. 14: 133-138.

2. Alpert, N. R. 1971. Preface. Cardiac Hypertrophy. N. R. Alpert, editor. Academic Press, Inc. New York. XVXVI.

3. Linzbach, A. J. 1960. Heart failure from the point of view of quantitative anatomy. Am. J. Cardiol. 5: 370 382.

4. Grant, C., D. G. Greene, and I. L. Bunnell. 1965. Left ventricular enlargement and hypertrophy. A clinical angiocardiographic study. Am. J. Mcd. 39: 895-904.

5. Levine, N. D., S. D. Rockoff, and E. Braunwald. 1963. An angiocardiographic analysis of the thickness of the left ventricular wall and cavity in aortic stenosis and other valvular lesions. Circulation. 28: 339-345.

6. Henry, W. L., C. E. Clark, and S. E. Epstein. 1973. Asymmetric septal hypertrophy (ASH): the unifying link in the IHSS disease spectrum. Circulation. $47: 827-$ 832.

7. Meerson, F. Z. 1969. The myocardium in hyperfunction, hypertrophy and heart failure. Circ. Res. 25 (Suppl. $I I): 1-163$.

8. Fanburg, B. L. 1970. Experimental cardiac hypertrophy. N. Engl. J. Med. 282: 723-732.

9. Peterson, M. B., and M. Lesch. 1972. Protein synthesis and amino acid transport in the isolated rabbit right ventricular papillary muscle. Circ. Res. 31: 317-327.

10. Hjalmarson, A., and O. Isaksson. 1972. In vitro work load and rate heart metabolism. I. Effect on protein synthesis. Acta. Physiol. Scand. 86: 126-144.

11. Spotnitz, H. M., and E. H. Sonnenblick. 1973. Structural conditions in the hypertrophied and failing heart. Am. J. Cardiol. 32: 398-406.

12. Grossman, W. 1974. Pressure measurement. In Cardiac Catheterization and Angiography. W. Grossman, editor. Lea \& Febiger, Philadelphia. 73-82.

13. McLaurin, L. P., W. Grossman, M. A. Stefadouros, E. L. Rolett, and D. T. Young. 1973. A new technique for the study of left ventricular pressure-volume relations in man. Circulation. 48: 56-64.

14. Grossman, W., M. A. Stefadouros, L. P. McLaurin, E. L. Rolett, and D. T. Young. 1973. The quantitative assessment of left ventricular diastolic stiffness in man. Circulation. $47: 567-574$

15. Grossman, W., L. P. McLaurin, S. P. Moos, M. A. Stefadouros, and D. T. Young. 1974. Wall thickness and diastolic properties of the left ventricle. Circulation. 49 : 129-135.

16. Grossman, W., L. P. McLaurin, and M. A. Stefadouros. 1974. Left ventricular stiffness in pressure and volume overload states in man. Circ. Res. 35: 793-800.

17. Popp, R. L., S. B. Wolfe, T. Hirata, and H. Feigenbaum. 1969. Estimation of right and left ventricular size by ultrasound. Am. J. Cardiol. 24: 523-530.

18. Pombo, J. F., B. L. Troy, and R. O. Russell, Jr. 1971. Left ventricular volumes and ejection fraction by echocardiography. Circulation. 43: 480-490.

19. Feigenbaum, H., R. L. Popp, S. B. Wolfe, B. L. Troy, J. F. Pombo, C. L. Haine, and H. T. Dodge. 1972. Ultrasound measurements of the left ventricle: a correlative study with angiocardiography. Arch. Intern. Med. 129: 461-467.

20. Murray, J. A., W. Johnston, and R. M. Reid. 1972. Echocardiographic determination of left ventricular dimensions, volumes, and performance. Am. J. Cardiol. $30: 252-257$.

21. Popp, R. L., and D. C. Harrison. 1970. Ultrasonic cardiac echocardiography for determining stroke volume and valvular regurgitation. Circulation. 41: 493-502.

22. Fortuin, N. J., W. P. Hood Jr., M. E. Sherman, and E. Craige. 1971. Determination of left ventricular volumes by ultrasound. Circulation. 44: 575-584.

23. Martin, H. A., and J. P. Murgo. 1973. Continuous left ventricular volume, circumferential fiber shortening rate and pressure volume loops by echocardiography and a multisensor catheter. Circulation. 48: IV-48.

24. Troy, B. L., J. Pombo, and C. E. Rackley. 1972. Measurement of left ventricular wall thickness and mass by echocardiography. Circulation. 45: 602-611.

25. Walker, M. L., Jr., E. W. Hawthorne, and H. Sandler. 1971. Methods for assessing performance for the intact hypertrophied heart. In Cardiac Hypertrophy. N. R. Alpert, editor. Academic Press, Inc., New York. 387405. 
26. Remington, R. D., and M. A. Schork. 1970. Statistics with Applications to the Biological Sciences. PrenticeHall, Inc., Englewood Cliffs, N. J.

27. Brodie, B. R., W. Grossman, and L. P. McLaurin. 1975. Combined hemodynamic-ultrasonic method for studying left ventricular wall stress: comparison with angiography. Am. J. Cardiol. 35: 124. (Abstr.)

28. Sandler, H., and H. T. Dodge. 1963. Left ventricular tension and stress in man. Circ. Res. 13: 91-104.

29. Hood, W. P., Jr., C. E. Rackley, and E. L. Rolett. 1968. Wall stress in the normal and hypertrophied human left ventricle. Am. J. Cardiol. 22: 550-558.

30. Hood, W. P., Jr. 1971. Dynamics of hypertrophy in the left ventricular wall of man. In Cardiac Hypertrophy.
N. R. Alpert, editor. Academic Press, Inc., New York. 445-452.

31. Legato, M. J. 1970. Sarcomerogenesis in human myocardium. J. Mol. Cell. Cardiol. 1: 425-437.

32. Falsetti, H. L., R. E. Mates, C. Grant, D. G. Greene, and I. L. Bunnell. 1970. Left ventricular wall stress calculated from one-plane cineangiography: an approach to force-velocity analysis in man. Circ. Res. 26: 71-83.

33. Bishop, V. S., and L. D. Horwitz. 1970. Left ventricular transverse internal diameter: value in studying ventricular function. Am. Heart J. 80: 507-514.

34. Ratshin, R. A., C. E. Rackley, and R. O. Russell, Jr. 1974. Determination of left ventricular preload and afterload by quantitative echocardiography in man. Circ. Res. 34: 711-718. 